\title{
Procedimientos de exploración y valores de referencia del rango de movimiento de la abducción de la cadera en deportistas. ¿Una herramienta en la prevención de lesiones?
}

\section{Scanning procedures and reference values in the range of motion of the hip abduction in athletes. A tool for the prevention of injuries?}

\author{
Cejudo, A. ${ }^{1}$, Sainz de Baranda, P. ${ }^{2 *}$, Santonja, F. ${ }^{3}$ y Ayala, F. ${ }^{4}$ \\ ${ }^{1}$ Centro Deportivo INACUA. Murcia, España. Doctorando en Ciencias de la Actividad Física y del Deporte. \\ Facultad de Ciencias del Deporte. Universidad de Murcia, España. \\ ${ }^{2}$ Facultad de Ciencias del Deporte. Universidad de Murcia, España. \\ ${ }^{3}$ Facultad de Medicina. Universidad de Murcia. Hospital Universitario. V. de la Arrixaca de Murcia, España. \\ ${ }^{4}$ Centro de Investigación del Deporte. Universidad Miguel Hernández de Elche, España.
}

\begin{abstract}
Resumen: El rango de movimiento limitado de la abducción de la cadera por una menor extensibilidad o cortedad de los aductores predispone a ciertas lesiones deportivas. Las pruebas angulares de la Abducción de la Cadera con Pierna Recta $\left(\mathrm{AB}_{\mathrm{PR}}\right)$ y la Abducción de la Cadera en Flexión $\left(A B_{F}\right)$ deben incluirse en cualquier evaluación deportiva músculo-esquelética. La utilización de ambas pruebas exploratorias permitirá evaluar específicamente la extensibilidad aductora. Para la medición del rango será necesario el uso de un goniómetro de rama larga y de un inclinómetro con varilla telescópica. De manera general, se han encontrado elevados valores de fiabilidad relativa y absoluta en el test $\mathrm{AB}_{\mathrm{PR}}$. Por el contrario, no se han encontrado estudios que analicen la fiabilidad del test $A_{\mathrm{F}}$.

Palabras claves: rango de movimiento, flexibilidad, abducción de la cadera, deporte.
\end{abstract}

Abstract: The limited range of motion of the hip abduction by lower extensibility or tightness adductor predisposes certain sports injuries. The angular testing of Hip Abduction Straight Leg $\left(\mathrm{HA}_{\mathrm{SL}}\right)$ and Hip Abduction in Flexion $\left(\mathrm{HA}_{\mathrm{F}}\right)$ should be included in any sport musculoskeletal assessment. Using both screening tests specifically will assess the adductor extensibility. For the measurement range will require the use of a long arm goniometer and an inclinometer with a telescopic arm. In general, they found elevated levels of relative and absolute reliability in the test $\mathrm{HA}_{\mathrm{SL}}$. Conversely, they have not found studies analyzing $\mathrm{HA}_{\mathrm{F}}$ reliability test.

Keywords: range of motion, flexibility, hip abduction, sport.

\section{Introducción}

Los músculos aductores de la cadera se agrupan en cinco músculos (pectíneo, aductor menor, aductor mediano o largo, aductor mayor y recto interno o gracilis) que ocupan la parte interna del muslo. El recto interno, a diferencia de los otros músculos, es biarticular, salta la cadera y la rodilla insertándose en la tibia formando parte de la pata de ganso (CalaisGermain, 2006).

Los aductores juegan un papel importante en el movimiento y en la estabilidad de la cadera durante las acciones técnicas del deporte. La cortedad de los aductores restringe el rango de movimiento "Range of Motion" (ROM), lo que predispone a ciertas lesiones deportivas como la osteopatía de pubis (Anderson, Strickland \& Warren, 2001; Verrall Hamilton, Slavotinek, Oakeshott, Spriggins, Barnesb \& Fon, 2005) o la rotura muscular (Ekstrand \& Gillquist, 1983; Ekstrand \& Hilding, 1999; Arnason, Sigurdsson, Gudmundsson, Holme, Engebretsen \& Bahr, 2004; Witvrouw, Danneels, Asselman, D’Have \& Cam-

Dirección para correspondencia [Correspodence address]: P. Sainz de Baranda. E-mail: psainzdebaranda@um.es bier, 2003; Verrall, Slavotinek, Barnes, Esterman, Oakeshott \& Spriggins, 2007). Estas lesiones son relativamente frecuentes en deportes como el fútbol (Anderson, Strickland \& Warren, 2001; Arnason et al., 2004; Egebretsen, Myklebust, Holme, Egebretsen \& Bahr, 2010; Wong \& Hong, 2005), fútbol australiano (Verrall, Slavotinek, Barnes, Esterman, Oakeshott \& Spriggins, 2007), tenis (Anderson, Strickland \& Warren, 2001; Young, Dakic, Stroia, Nguyen, Harris \& Safran, 2014), baloncesto (Dallinga, Benjaminse \& Lemmink, 2012), voleibol (Dallinga, Benjamins \& Lemmink, 2012), natación (Grote, Lincoln \& Gamble, 2004), carrera de fondo con o sin obstáculos (Verrall, Hamilton, Slavotinek, Oakeshott, Spriggins, Barnes \& Fon, 2005) y hockey hielo (Anderson, Strickland \& Warren, 2001; Emery \& Meeuwisse, 2001).

La capacidad de predecir estas lesiones deportivas es tan importante como el evaluarlas y tratarlas. Por ello, la medición de la extensibilidad de los aductores de la cadera ha de ser incluida en cualquier programa preventivo con el objetivo de identificar la cortedad, al ser ésta un factor de riesgo intrínseco modificable (Witvrouw, Bellemans, Lysens, Danneels 
\& Cambier, 2001; Malliaras, Cook \& Kent, 2006; Bradley $\&$ Portas, 2007). También, se aconseja valorar la flexibilidad durante toda la temporada con el objetivo de realizar una planificación real y ajustada a los valores específicos de cada deportista (Irurtia, Busquets, Carrasco, Ferrer \& Marina, 2010). Así, el diseño ideal de un programa físico-técnico deportivo, incluye la valoración inicial de la flexibilidad en la pretemporada (Knapik, Bauman, Jones, Harri \& Vaughan, 1991; Arnason, Gudmundsson, Dahl \& Johannsson, 1996) y la realización de un control o seguimiento durante la temporada deportiva (Irurtia et al., 2010; Frehill, Brian, Archer, Bancells, Wilckens, McFarland \& Cosgarea, 2011).

Los objetivos principales de esta revisión bibliográfica son: a) describir la metodología de valoración de las pruebas angulares más empleadas en el ámbito clínico y científico para medir la extensibilidad aductora; así como b) aportar valores de referencia que puedan ser utilizados por los profesionales del deporte y de la salud para categorizar la flexibilidad de los aductores de sus deportistas.

\section{Pruebas para valorar el rango de movimiento de la abducción de la cadera}

La extensibilidad de los aductores se mide indirectamente mediante el ROM de la abducción de la cadera (Weppler \& Magnusson, 2010). En la bibliografía se describen diferentes procedimientos que utilizan diversos instrumentos de valoración como el goniómetro (Alricsson \& Werner 2004; Steinberg, Hershkovitz, Peleg, Dar, Masharawi, Heim \& Siev-Ner, 2006), el hidrogoniómetro (Gannon \& Bird, 1999), el inclinómetro (Gerhardt, 1994; Gerhardt, Cocchiarella \& Lea, 2002; Cejudo, Sainz de Baranda, Ayala \& Santonja, 2015) o el análisis cinemático (Arnason et al., 2004; Bozic, Pazin, Berjan, Planic, \& Cuk, 2010; Fourchet, Materne, Horobeanu, Hudacek \& Buchheit, 2013). Se encuentran propuestas donde el movimiento es generado por un operador (manual o mecánico -dinamómetro, tensiometro-) (Witvrouw et al., 2003; Arnason et al., 2004; Fourchet et al, 2013) o propuestas donde el movimiento es realizado de forma activa por el propio explorado (Gannon \& Bird, 1999; Bozic et at., 2010).

Para valorar la extensibilidad de los aductores, tanto los monoarticulares (pectíneo, aductor menor, aductor mediano y aductor mayor) como el biarticular (recto interno), la prueba angular mas utilizada ha sido la abducción de cadera con la pierna recta $\left(\mathrm{AB}_{\mathrm{PR}}\right)$ (Norkin \& White, 1995; Clarkson, 2003). Esta prueba ha sido descrita en tres posiciones iniciales utilizando para su valoración, en la mayoría de las ocasiones, un goniómetro de rama larga.

La primera propuesta, parte con el explorado tumbado en una camilla en posición decúbito supino con ambas extremidades inferiores en posición neutra para evitar la abducción de la extremidad contralateral (American Academia of Or- thopaedic Surgeons [AAOS], 1965; Norkin \& White, 1995, Palmer \& Epler, 2002) (figura 1).

Figura 1. Valoración del ROM de la abducción de la cadera con ambas caderas en posición neutra (AAOS, 1965).

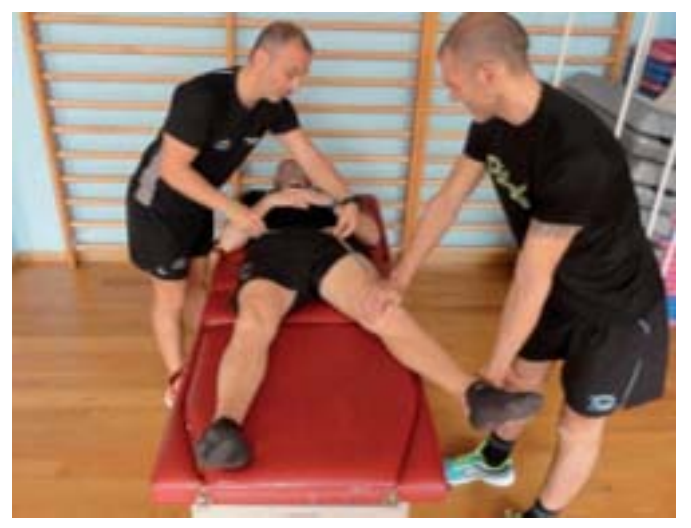

La segunda propuesta, parte con el explorado tumbado en una camilla en posición decúbito supino, pero con la cadera contralateral en ligera abducción y la pierna en flexión colgando por el lateral de la camilla para fijar la pelvis (Reid, Burnham, Saboe \& Kushner 1987; Ekstrand, Wiktorsson, Oberg \& Gillquist, 1982; Clarkson, 2003; Alricsson \& Werner, 2004; Witvrouw et al., 2003; Zakas, 2005; Fourchet et al, 2013; Nussbaumer, Leunig, Glatthorn, Stauffacher, Gerber \& Maffiuletti, 2010; Cejudo et al., 2015) (figura 2).

Figura 2. Valoración del ROM de la abducción de cadera con la pierna recta con la pierna de la extremidad no evaluada colgando por el lateral de la camilla.

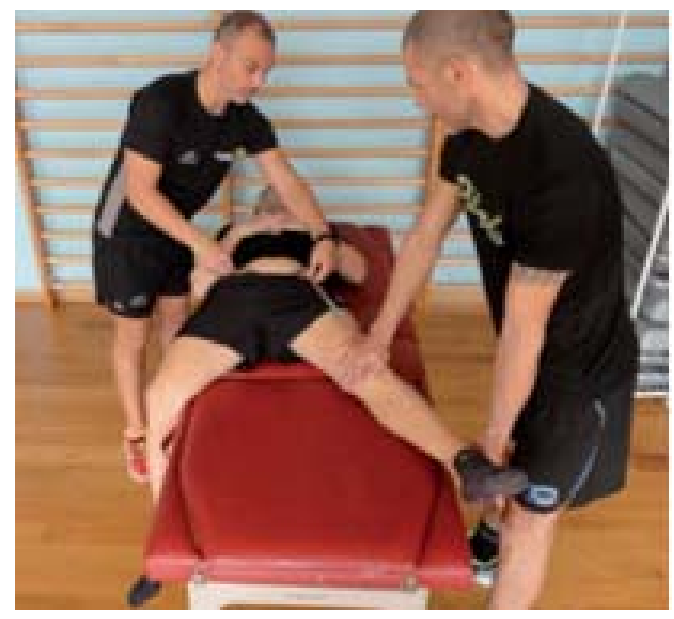

La tercera propuesta parte con el explorado tumbado en una camilla en posición decúbito lateral y utiliza como instrumento de valoración el inclinómetro (Gerhardt, 1994; Gerhardt et al., 2002) (figura 3). 
Figura 3. Valoración del ROM de la abducción de la cadera con la pierna recta en decúbito lateral.

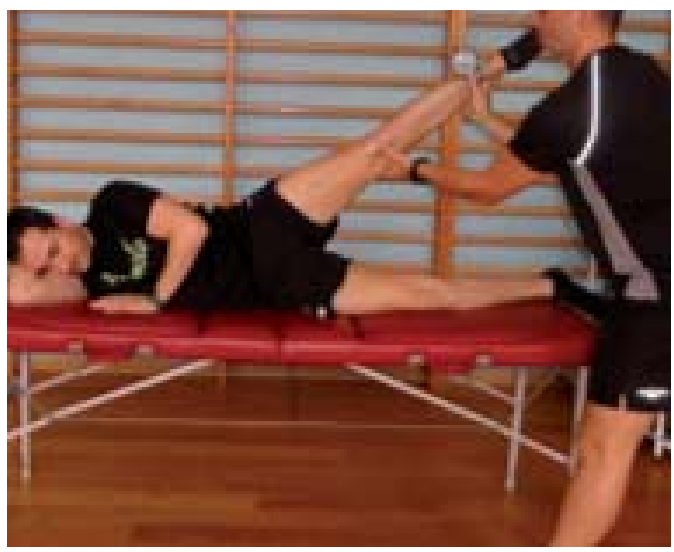

Por otro lado, para valorar específicamente la extensibilidad de los aductores monoarticulares, sin implicar al recto interno, ha sido descrito la prueba de ROM de abducción de la cadera en flexión (prueba $\mathrm{AB}_{\mathrm{F}}$ ) (AAOS, 1965; Gerhardt, 1994; Cejudo, Ayala, Sainz de Baranda \& Santonja, 2015b). En este caso, el explorado se coloca en decúbito supino con la cadera y la rodilla explorada a $90^{\circ}$ de flexión, y para la posición de la extremidad no explorada se describen tres opciones: a) con la cadera y rodilla a 90 de flexión (AAOS, 1965; Alter, 2004), b) con la pierna colgando por el frontal de la camilla (Gerhardt, 1994) y c) en posición neutra. En este caso el ángulo de abducción de cadera puede medirse con goniómetro o con inclinómetro con varilla telescópica extensible (figura 4).

Figura 4. Valoración del ROM de la abducción de la cadera en flexión.
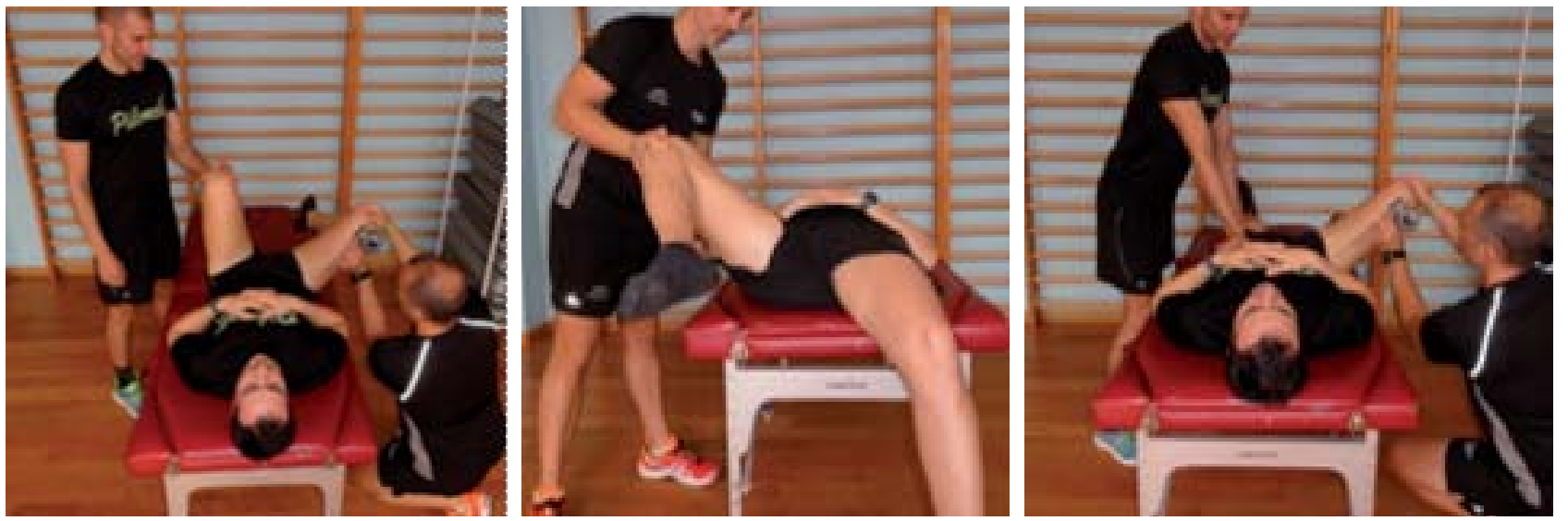

\section{Valores de referencia}

La detección de la cortedad muscular de un deportista, debe ser determinada mediante la comparación de la medición del ROM de la abducción de la cadera con los valores de referencia del deporte practicado y atendiendo a la especificidad de esta cualidad física básica (Cejudo et al., 2014a,b). Desafortunadamente, los resultados publicados sobre el ROM de abducción de cadera en el deporte son escasos. La mayoría de los valores de referencia utilizados son los publicados por la American Academia of Orthopaedic Surgeons (1965), la American Medical Association [AMA] (Gerhardt et al., 2002) y los propuestos en algunos libros de texto de valoración músculo-esquelética
(Gerhardt, 1994; Norkin \& White, 1995, Palmer \& Epler, 2002; Clarkson, 2003; Taboadela, 2007) (tabla 1).

En la literatura científica existen diferentes estudios que han valorado la flexibilidad de los aductores en deportistas de diferentes niveles competitivos. En la tabla 2 se muestran los valores medios obtenidos en diferentes deportes tras la valoración de la abducción de cadera con la pierna recta (prueba $\left.\mathrm{AB}_{\mathrm{PR}}\right)$. Todos estos trabajos valoran la abducción de la cadera desde la posición neutra con la pierna de la extremidad no evaluada colgando por el lateral de la camilla. Los valores medios se encuentran en un rango entre los $33,5^{\circ}$ en futbolistas sénior (Ekstrand \& Gillquist, 1982) y los 57,30 en jugadores de fútbol sala (Cejudo, Sainz de Baranda, Ayala \& Santonja, 2014a). 
Tabla 1. Revisión de los rangos de valores de normalidad publicados en población general.

\begin{tabular}{|c|c|c|c|}
\hline \multicolumn{2}{|r|}{ Posición inicial } & \multirow{2}{*}{ Referencia } & \multirow{2}{*}{ Rango normal } \\
\hline Extremidad explorada & Extremidad contralateral & & \\
\hline \multirow{6}{*}{$\begin{array}{l}\text { Decúbito supino } \\
\left(\text { cadera y rodilla a } 0^{\circ}\right)\end{array}$} & \multirow{5}{*}{ Posición neutra. No se fija con la camilla } & AAOS (1965) & $0^{\circ}$ a $45^{\circ}$ \\
\hline & & Palmer \& Epler (2002) & $0^{\circ}$ a $45^{\circ}$ \\
\hline & & Alter (2004) & $0^{\circ}$ a $45^{\circ}$ \\
\hline & & Norkin \& White (1995) & AAOS \\
\hline & & Taboadela (2007) & AAOS \\
\hline & $\begin{array}{l}\text { Levemente abducida y la pierna colgando por } \\
\text { fuera de la camilla para fijar la pelvis }\end{array}$ & Clarkson (2003) & $0^{\circ}$ a $45^{\circ}$ \\
\hline \multirow{4}{*}{$\begin{array}{l}\text { Decúbito supino, abduc- } \\
\text { ción en flexión (cadera a } \\
90^{\circ} \text {, rodilla flexionada). }\end{array}$} & \multirow{2}{*}{ Cadera y rodilla no explorada a $0^{\circ}$} & Gerhardt (1994) & $0^{\circ}$ a $50^{\circ}$ \\
\hline & & Debruner SUVA citado en Gerhardt (1994) & $0^{\circ}$ a $80^{\circ}$ \\
\hline & \multirow{2}{*}{ Cadera y rodilla no explorada a $90^{\circ}$} & AAOS (1965) & $45^{\circ}$ a $60^{\circ}$ \\
\hline & & Alter (2004) & No detalla \\
\hline \multirow{2}{*}{$\begin{array}{l}\text { Decúbito lateral } \\
\text { (cadera y rodilla a } 0^{\circ} \text { ) }\end{array}$} & \multirow{2}{*}{ Posición neutra } & Gerhardt (1994) & $0^{\circ}$ a $50^{\circ}$ \\
\hline & & Gerhardt et al. [AMA] (2002) & $0^{\circ}$ a $40^{\circ}\left(45^{\circ}\right)$ \\
\hline
\end{tabular}

Para el ROM de la abducción de cadera en flexión (prueba $\mathrm{AB}_{\mathrm{F}}$ ) no se han encontrados valores publicados en población deportista. Sin embargo, nuestro grupo de investigación tras utilizar esta prueba en varios estudios ha encontrado un rango cifrado entre $59,3^{\circ}$ y $75,1^{\circ}$ en jugadores de fútbol sala y de baloncesto federado (datos no publicados).

Tabla 2. Valores de referencia obtenidos en diferentes deportes tras la valoración de la abducción de cadera.

\begin{tabular}{|c|c|c|c|c|c|}
\hline $\begin{array}{l}\text { Zakas et al. (2002) } \\
\text { Balonmano junior } \\
\qquad \mathrm{H}(\mathrm{n}=20)\end{array}$ & $46,4^{\circ}$ & $\begin{array}{c}\text { Zakas et al. (2003) } \\
\text { Balonmano junior } \\
\text { H (n=47) }\end{array}$ & $45,3^{\circ}$ & $\begin{array}{c}\text { Cejudo et al. (2014b) } \\
\text { Balonmano } 1^{\text {a }} \text { DNE } \\
\text { H }(\mathrm{n}=22)\end{array}$ & $46^{\circ}$ \\
\hline $\begin{array}{c}\text { Cejudo et al. (2014b) } \\
\text { Balonmano 2a DNE Nacional } \\
\text { H }(\mathrm{n}=22)\end{array}$ & $48,5^{\circ}$ & $\begin{array}{l}\text { Cejudo y cols. (2014a) } \\
\text { Fútbol sala 2a DNE } \\
\text { H }(n=17)\end{array}$ & $51,7^{\circ}$ & $\begin{array}{c}\text { Cejudo y cols. (2014a) } \\
\text { Fútbol sala 2a DNE Porteros } \\
\text { H }(n=3)\end{array}$ & $57,3^{\circ}$ \\
\hline $\begin{array}{c}\text { Ekstrand \& Gillquist (1982) } \\
\text { Fútbol senior } \\
\text { H }(\mathrm{n}=180)\end{array}$ & $33,5^{\circ}$ & $\begin{array}{l}\text { Witvrouw et al. (2003) } \\
\text { Fútbol profesional } \\
\text { H }(\mathrm{n}=146)\end{array}$ & $53,3^{\circ}$ & $\begin{array}{c}\text { Arnason et al. (2004) } \\
\text { Fútbol elite } \\
\text { H }(\mathrm{n}=153)\end{array}$ & $43,4^{\circ}$ \\
\hline $\begin{array}{l}\text { Zakas \& cols. }(2005) \\
\text { Fútbol adolescente } \\
\text { H }(\mathrm{n}=50)\end{array}$ & $46,3^{\circ}$ & $\begin{array}{l}\text { Zakas et al. (2006) } \\
\text { Fútbol adolescente } \\
\qquad \mathrm{H}(\mathrm{n}=80)\end{array}$ & $39,8^{\circ}$ & $\begin{array}{c}\text { Manning \& Hudson (2009) } \\
\text { Fútbol profesional } \\
\text { H }(\mathrm{n}=20)\end{array}$ & $50^{\circ}$ \\
\hline $\begin{array}{c}\text { Manning \& Hudson (2009) } \\
\text { Fútbol profesional } \\
16-18 \text { ańos } \\
\mathrm{H}(\mathrm{n}=20)\end{array}$ & $55^{\circ}$ & $\begin{array}{c}\text { Cejudo et al. (2012) } \\
\text { Fútbol 3a DNE } \\
\text { H }(n=22)\end{array}$ & $41,1^{\circ}$ & $\begin{array}{c}\text { Cejudo et al. (2013) } \\
\text { Duatlón senior } \\
\text { H (n=15) }\end{array}$ & $50,7^{\circ}$ \\
\hline $\begin{array}{c}\text { Tyler et al. (2001) } \\
\text { Hockey hielo profesional } \\
\text { H (n=47) }\end{array}$ & $45,8^{\circ}$ & $\begin{array}{c}\text { Zakas (2005) } \\
\text { Tenis junior elite } \\
\mathrm{H}(\mathrm{n}=24)\end{array}$ & $41,6^{\circ}$ & $\begin{array}{c}\text { Sainz de Baranda et al. (2015) } \\
\text { Fútbol sala elite } \\
\text { M (n=15) }\end{array}$ & $45,2^{\circ}$ \\
\hline
\end{tabular}

H: Hombres; M: Mujeres; DNE: División Nacional Española.

Procedimiento de valoración del rango de movimiento de la abducción de la cadera

En el ámbito deportivo, para medir la extensibilidad de los aductores deben utilizarse dos pruebas de valoración: a) el
ROM de la abducción de la cadera (prueba $\mathrm{AB}_{\mathrm{PR}}$ ) y el ROM de la abducción de la cadera en flexión (prueba $\mathrm{AB}_{\mathrm{F}}$ ). Ambas pruebas son necesarias si se quiere diferenciar la extensibilidad de los aductores. Para la correcta ejecución de estas dos pruebas será preciso tener en cuenta algunas consideraciones 
sobre el procedimiento exploratorio, el instrumento de medición, el calentamiento, posición final, la validez, la fiabilidad $\mathrm{y}$ valores de referencia.

\section{Procedimiento exploratorio}

Prueba angular de abducción de la cadera con pierna recta (test APR)

Los diferentes manuales de valoración músculo-esquelética (AAOS, 1965; Norkin \& White, 1995; Palmer \& Epler, 2002) proponen que el explorado se coloque tumbado en decúbito supino en posición neutra "Standardized Neutral Zero Measuring" (Gerhardt et al., 2002) y que se inicie el movimiento de abducción con ambas caderas en posición neutra. Sin embargo, algunos autores plantean la opción de que el explorado coloque la pierna no explorada colgando por el lateral de la camilla debido a que con esta posición inicial es más fácil la fijación de la pelvis al tener un punto fijo (Reid et al., 1987; Ekstrand et al., 1982; Clarkson, 2003; Alricsson \& Werner, 2004; Witvrouw et al., 2003; Zakas, 2005; Fourchet et al, 2013; Nussbaumer et al., 2010; Cejudo et al., 2015). La posición inicial elegida no va a condicionar el rango puro de abducción por posibles movimientos compensatorios (abducción de la cadera contralateral e inclinación lateral de la pelvis), ya que éste se obtiene entre la línea que une ambas espinas iliacas ántero-superiores y la bisectriz de la cara anterior del muslo. Sin embargo, la opción que parte con la pierna no explorada colgando por el lateral de la camilla permite tener un punto fijo, lo cual es recomendado por algunos autores al facilitar la medición de la extensibilidad máxima (Clarkson, 2003; Palmer \& Epler, 2002) (apéndice 1).

El ROM de la abducción de la cadera puede incrementarse cuando se permite que el explorado realice una rotación externa de cadera, debido a que al relajar el ligamento isquio-femoral, se coloca la pelvis en anteversión y la columna lumbar en hiperextensión (Alter, 2004). Este movimiento suele utilizarse en determinados gestos técnicos de la danza y en el ballet. Para estas disciplinas se ha descrito la medición del ROM de la abducción de la cadera en rotación externa para simular las exigencias del gesto técnico [perspectiva del rendimiento técnico] (Alricsson, Harms-Ringdahl, Eriksson \& Werner, 2003; Alricsson \& Werner, 2004). Sin embargo, para medir la abducción de la cadera pura (Gerhardt et al., 2002) debe detenerse la prueba cuando el examinador auxiliar detecte cualquiera de los movimientos de compensación incluyendo la rotación externa de cadera (apéndice 1).

La posición en decúbito lateral no es recomendable debido a que presenta una serie de limitaciones importantes, al no haber sido definida la posición $0^{\circ}$ en decúbito lateral, por la dificultad de estabilizar la pelvis en posición neutra y de controlar los movimientos compensatorios.
Prueba angular abducción de la cadera en flexión (test $\mathrm{AB}_{\mathrm{F}}$ )

Es recomendable incluir esta prueba de valoración debido a la especificidad de su medida (aductores monoarticulares). La limitación de la abducción de la cadera en flexión se deberá fundamentalmente a los músculos y en menor medida a los ligamentos. Por ello, se observa un mayor ROM con la cadera y rodilla en flexión, debido a que al flexionar la cadera se relajan los ligamentos isquio-femoral, pubo-femoral e ilio-femoral (Kapandji, 2007), y a que el recto interno no interviene como limitador del movimiento al flexionarse la rodilla. Si existiese una gran extensibilidad músculo-tendinosa el movimiento podría ser restringido por el contacto entre el cuello del fémur y la ceja cotiloidea. Si la abducción se realiza en posición anatómica, la tensión de los ligamentos puede limitar el movimiento antes que los músculos.

En relación a la posición inicial, se han encontrado dos propuestas para la posición de la extremidad no explorada: 1) con la cadera y rodilla flexionada a $90^{\circ}$ y 2) con la cadera y rodilla en posición neutra. Parece evidente que la segunda propuesta aporta una mayor estabilidad y facilidad a la hora de fijar la pelvis y controlar las compensaciones (apéndice 2).

\section{Instrumento de medida}

El goniómetro es uno de los instrumentos más utilizados para medir los ángulos en el sistema osteoarticular. Sin embargo, el uso del inclinómetro parece más recomendable debido a que la varilla telescópica hace que éste se convierta en un goniómetro de un sólo brazo, con la ventaja de tener un indicador de gravedad que permite una mayor precisión y reproducibilidad de las medidas, además de incrementar la velocidad de medición (Gerhardt, 1994; Gerhardt et al., 2002). Sin embargo, habrá que tener en cuenta que el inclinómetro no podrá utilizarse en la prueba $A_{\mathrm{PR}}$ ya que depende de la fuerza de gravedad para su funcionamiento.

\section{Calentamiento}

Durante la sesión de valoración, y previo a la aplicación de las diferentes pruebas exploratorias del ROM pasivo máximo, se recomienda realizar ejercicio aeróbico entre 8-10 minutos (intensidad ligera [10-12 escala Borg]) unido 2 series de 30 segundos de estiramientos estáticos activos estandarizados, enfatizando la actividad de los músculos aductores, bajo la estricta supervisión de los examinadores. La intensidad y duración del calentamiento aeróbico se basa en las recomendaciones establecidas por Bishop (2003), quien tras efectuar una profunda revisión bibliográfica sobre el calentamiento concluye que para maximizar el incremento de la temperatura muscular y optimizar el rendimiento posterior es necesario ejecutar acciones de movilidad global (principalmente carre- 
ra) durante aproximadamente 10 minutos a una intensidad del 40-60\% VO2máx. Igualmente, se recomienda aplicar un volumen total de 60 segundos (2 series de 30 segundos) por grupo muscular y lado corporal asegurar la estabilidad de las propiedades viscoelásticas (Cejudo et al., 2015).

\section{Posición final de valoración}

El resultado final de cada intento se determina por uno o varios de los siguientes criterios: (1) el examinador percibia resistencia firme por el músculos estirados (AAOS, 1965; Zakas, 2005; Aalto, Airaksinen, Härkönen \& Arokoski, 2005); (2) el explorado sentía estiramiento muscular fuerte pero tolerable (Ekstrand et al., 1982; Tyler, Nicholas, Campbell \& McHugh, 2001; Witvrouw et al., 2003; Zakas et al., 2003); (3) los examinadores detectaba algún movimiento de compensación (rotación de la cadera e incremento de la lordosis lumbar) que incrementaba el ROM (Ekstrand et al.,1982; Clarkson, 2003; Cejudo et al, 2015) o (4) por la aparición de algias en la articulación explorada.

Validez de la medida

La validez de una prueba de evaluación es definida como el grado de precisión con el cual ésta mide exactamente aquello que se ha propuesto medir, es decir, el grado con el cual se cumple su objetivo (Hopkins, 2000). En este sentido, ambas pruebas han sido considerados válidos por las más prestigiosas organizaciones americanas de medicina (AAOS, 1965; AMA, 2001) e incluidas en los manuales más acreditados del área de la medicina y ciencias del deporte (Magee, 2002; Palmer \& Epler, 2002; Clarkson, 2003; Peterson, Kendall \& Geise, 2005), basándose en los conocimientos anatómicos, en las habilidades y en una amplia experiencia práctica.

\section{Fiabilidad de la medida}

Pocos estudios han valorado la fiabilidad intra-sesión y la fiabilidad inter-sesión de la prueba de la abducción de la cadera con la pierna recta, concretamente 5 estudios han valorado la fiabilidad intra-sesión y 7 estudios la fiabilidad inter-sesión.

La prueba $A_{P R}$ ha demostrado poseer de forma generalizada una elevada fiabilidad relativa o intra-sesión, mostrando valores de coeficiente de variación entre el 1,4\% y el $10 \%$ y un Índice de Correlación Intraclase superior a 0,85 (tabla 1).

Tabla 1. Fiabilidad intra-sesión del ROM de la abducción de la cadera con la pierna recta (prueba $\mathrm{AB}_{\mathrm{PR}}$ ).

\begin{tabular}{|c|c|c|c|c|}
\hline Referencia Muestra & Recursos humanos y materiales & Procedimiento exploratorio & $\begin{array}{c}\text { No sesiones e intervalo } \\
\text { de tiempo }\end{array}$ & Resultados \\
\hline $\begin{array}{c}\text { Ekstrand et al. (1982) } \\
\mathrm{H}(\mathrm{n}=12) \\
\text { Adultos jóvenes } \\
\end{array}$ & $\begin{array}{l}\text { Dos examinadores } \\
\text { Flexómetro Myrin }\end{array}$ & $\begin{array}{l}\text { No calentamiento } \\
\text { Un intento }\end{array}$ & $\begin{array}{c}1 \text { sesión } \\
\text { No detalla }\end{array}$ & $C V=1,4 \pm 0,8 \%$ \\
\hline $\begin{array}{c}\text { Arnason et al. }(2004) \\
\text { No detalla }(\mathrm{n}=31) \\
\text { Deportistas }\end{array}$ & $\begin{array}{l}\text { No detalla } \\
\text { Goniómetro }\end{array}$ & No detalla & No detalla & $\mathrm{CV}=3,35 \%$ \\
\hline $\begin{array}{c}\text { Nussbaumer et al. (2010) } \\
\text { H }(\mathrm{n}=8) \\
\mathrm{M}(\mathrm{n}=7) \\
\text { Adultos patología de cadera }\end{array}$ & $\begin{array}{l}\text { Un examinador } \\
\text { Goniómetro }\end{array}$ & $\begin{array}{l}5 \text { min cicloergómetro } \\
2 \text { intentos y la media }\end{array}$ & $\begin{array}{c}1 \text { sesión } \\
\text { No detalla }\end{array}$ & $\mathrm{ICC}=0,92$ \\
\hline $\begin{array}{c}\text { Bozic et al (2010) } \\
\text { H ( } \mathrm{n}=8) \\
\text { Activos y deportistas }\end{array}$ & $\begin{array}{l}\text { Dos examinadores } \\
\text { Análisis cinemático }\end{array}$ & $\begin{array}{l}10 \text { min calentamiento } \\
10 \text { min de estiramiento }\end{array}$ & $\begin{array}{l}1 \text { sesión } \\
\text { No detalla }\end{array}$ & $\begin{array}{l}\mathrm{CV}=2,0 \% \\
\mathrm{ICC}=0,96\end{array}$ \\
\hline $\begin{array}{l}\text { Prather et al. (2010) } \\
\qquad \begin{array}{l}\mathrm{H}(\mathrm{n}=10) \\
\mathrm{M}(\mathrm{n}=18) \\
\text { Adultos sanos }\end{array}\end{array}$ & $\begin{array}{l}\text { Dos examinadores } \\
\text { Goniómetro }\end{array}$ & $\begin{array}{l}\text { No detalla } \\
3 \text { intentos }\end{array}$ & $\begin{array}{l}1 \text { sesión } \\
\text { No detalla }\end{array}$ & $\begin{array}{l}\mathrm{CV}=10 \% \\
\mathrm{ICC}=0,85\end{array}$ \\
\hline
\end{tabular}

H: Hombres; M: Mujeres; n= tamaño muestral; CV: Coeficiente de variación; ICC: Coeficiente de Correlación Intraclase.

La prueba $\mathrm{AB}_{\mathrm{PR}}$ presenta valores moderados-altos de fiabilidad absoluta o inter-sesión, mostrando valores de variabili- dad, en términos porcentuales en torno a $1,9 \%$ y $7,2 \%$ y en términos absolutos entre $1,8^{\circ}$ y $2,3^{\circ}$ (tabla 2 ). 
Tabla 2. Fiabilidad inter-sesión del ROM de la abducción de la cadera con la pierna recta (prueba $\mathrm{AB}_{\mathrm{PR}}$ ).

\begin{tabular}{|c|c|c|c|c|}
\hline Referencia Muestra & $\begin{array}{c}\text { Recursos humanos y } \\
\text { materiales }\end{array}$ & Procedimiento exploratorio & $\begin{array}{l}\text { No sesiones e interva- } \\
\text { lo de tiempo }\end{array}$ & Resultados \\
\hline $\begin{array}{c}\text { Ekstrand et al. (1982) } \\
\mathrm{H}(\mathrm{n}=12) \\
\text { Adultos jóvenes } \\
\end{array}$ & $\begin{array}{l}\text { Dos exploradores } \\
\text { Flexómetro Myrin }\end{array}$ & $\begin{array}{l}\text { No calentamiento } \\
\text { Un intento }\end{array}$ & $\begin{array}{l}2 \text { sesiones } \\
\text { No detalla }\end{array}$ & $C V=1,9 \pm 0,7 \%$ \\
\hline $\begin{array}{c}\text { Steinberg et al. }(2006) \\
\text { No detalla }(\mathrm{n}=20) \\
\text { Danza }\end{array}$ & $\begin{array}{l}\text { Dos exploradores } \\
\text { Goniómetro }\end{array}$ & No detalla & $\begin{array}{l}2 \text { sesiones } \\
1 \text { día }\end{array}$ & $\mathrm{ICC}=0,96$ \\
\hline $\begin{array}{l}\text { Reid et al. (1997) } \\
\text { M ( } \mathrm{n}=30) \\
\text { Estudiantes senior ballet }\end{array}$ & $\begin{array}{l}\text { Un examinador } \\
\text { Goniómetro }\end{array}$ & No detalla & $\begin{array}{l}3 \text { sesiones } \\
1 \text { día }\end{array}$ & $\mathrm{CV}=4,3 \%$ \\
\hline $\begin{array}{c}\text { Bozic et al (2010) } \\
\text { H }(\mathrm{n}=84) \\
\text { Activos y Deportistas }\end{array}$ & $\begin{array}{l}\text { Dos examinadores } \\
\text { Análisis cinemático }\end{array}$ & $\begin{array}{l}10 \text { min calentamiento } \\
10 \text { min de estiramiento }\end{array}$ & $\begin{array}{l}2 \text { sesiones } \\
1 \text { semana }\end{array}$ & $\begin{array}{l}\mathrm{CV}=3,4 \% \\
\mathrm{ICC}=0,87\end{array}$ \\
\hline $\begin{array}{c}\text { Cejudo et al. (2015) } \\
\mathrm{H}(\mathrm{n}=60)^{1,2} \\
\mathrm{M}(\mathrm{n}=30)^{1} \\
{ }^{1} \text { Futbol sala } \\
{ }^{2} \text { Balonmano } \\
\end{array}$ & $\begin{array}{l}\text { Dos examinadores } \\
\text { Inclinómetro } \\
\text { Lumbosant }\end{array}$ & $\begin{array}{c}5 \text { min jogging y estiramientos. } \\
2 \text { intentos y la media }\end{array}$ & $\begin{array}{l}3 \text { sesiones } \\
2 \text { semanas }\end{array}$ & $\begin{aligned} \mathrm{SEM} & =1,8^{\circ} \\
\mathrm{ICC} & =0,95\end{aligned}$ \\
\hline $\begin{array}{l}\text { Fourchet et al. (2013) } \\
\text { H (n=10) } \\
\text { Adolescentes deportistas de elite }\end{array}$ & $\begin{array}{l}\text { Un examinador } \\
\text { Cámaras de video }\end{array}$ & $\begin{array}{l}\text { No calentamiento } \\
1 \text { intento }\end{array}$ & $\begin{array}{l}2 \text { sesiones } \\
3 \text { días }\end{array}$ & $\begin{array}{r}\mathrm{CV}=7,2 \% \\
\mathrm{ICC}=0,85\end{array}$ \\
\hline $\begin{array}{c}\text { Nussbaumer et al. (2010) } \\
\text { H (n=8) } \\
M(n=7) \\
\text { Adultos patología de la cadera }\end{array}$ & $\begin{array}{l}\text { Un examinador } \\
\text { Goniómetro }\end{array}$ & $\begin{array}{l}5 \text { min cicloergómetro } \\
2 \text { intentos y la media }\end{array}$ & $\begin{array}{l}2 \text { sesiones } \\
1 \text { semana }\end{array}$ & $\begin{array}{l}\mathrm{SEM}=2,3^{\circ} \\
\mathrm{ICC}=0,92\end{array}$ \\
\hline $\begin{array}{l}\text { H: Hombres; M: Mujeres; } n=\text { tamańo mue } \\
\text { Sorprendentemente, de la prueba de } \mathrm{AB}_{\mathrm{F}} \mathrm{s} \\
\text { celentes valores de precisión de la medida, }\end{array}$ & $\begin{array}{l}\text { SEM: Error Estándar de la } 1 \\
\text { ha encontrado un estudio so } \\
\text { y } 81^{\circ} \text { empleando el Error Es }\end{array}$ & $\begin{array}{l}\text { ida; CV: Coeficiente de variación; ICC: } \\
\text { la fiabilidad (Cejudo, Ayala, Sainz de Bar } \\
\text { lar de la Medida [SEM] ( } 68 \% \text { probabilic }\end{array}$ & $\begin{array}{l}\text { oeficiente de Correlación Intra } \\
\text { anda, \& Santonja, 2015b). Este } \\
\text { ad) y Mínimo Cambio Detecta }\end{array}$ & $\begin{array}{l}\text { aclase. } \\
\text { e trabajo mostró ex- } \\
\text { able }\left[\mathrm{MDC}_{95}\right](95 \%\end{array}$ \\
\hline
\end{tabular}

\section{Valores de referencia en el deporte}

En el proceso de mejora del rendimiento deportivo, los preparadores y readaptadores físicos precisan valores de referencia de flexibilidad específicos de cada deporte (Palmer \& Epler, 2002; Cejudo et al., 2014a,b). La interpretación de la comparación ayudará a tomar decisiones en el diseño del entrenamiento con el propósito de mejorar el rendimiento físico-técnico deportivo. Por ello, son necesarios más estudios que valoren la flexibilidad en el deporte para poder definir el perfil de flexibilidad de cada deporte.

Tras la revisión bibliográfica, sólo se han encontrado cuatro trabajos que definen el perfil de flexibilidad en fútbol sala en ambos sexos, balonmano y duatlón (Cejudo et al., 2014 a, b y c; Sainz de Baranda et al., 2015). Por ello, en el presente trabajo se han recopilado los valores medios aportados en diferentes trabajos que han valorado la abducción de la cadera con la pierna recta en diferentes deportes. Los valores medios se encuentran en un rango que oscila entre los $33,5^{\circ}$ y los $57,3^{\circ}$.

Respecto a los valores de cortedad, solo Ekstrand \& Gillquist (1982), en una muestra de 180 jugadores sénior de fútbol, establecen el límite de cortedad en $28^{\circ}$ para la abducción de la cadera en posición neutra. Este punto de corte lo obtienen al correlacionar los valores de extensibilidad de los aductores con la lesión muscular del muslo y de la ingle.

La reducción del ROM de la abducción de la cadera es, junto a la asimetría entre el lado dominante y no dominante en los valores de extensibilidad, un factor de riesgo modificable en lesiones de las extremidades inferiores (Ellenbecker, Ellenbecker, Roetert, Silva, Keuter \& Sperling, 2007; Young, Dakic, Stroia, Nguyen, Harris, \& Safran, 2014). Por ello, será importante su evaluación para plantear de forma objetiva programas preventivos (Arnason et al., 2004).

Futuros estudios deben centrarse en definir el perfil de flexibilidad en cada deporte teniendo en cuenta el nivel competitivo, edad, sexo del deportista y atendiendo al puesto táctico. Además, es necesarioidentificar qué valor de extensibilidad aductora se correlaciona con la lesión deportiva. Sólo así, los preparadores y readaptadores físicos podrán establecer un trabajo de flexibilidad específico e individualizado para optimizar el rendimiento físico-técnico deportivo y minimizar el riesgo de lesión deportiva. 


\section{Conclusiones}

Las pruebas de la Abducción de la Cadera con Pierna Recta $\left(\mathrm{AB}_{\mathrm{PR}}\right)$ y Abducción de la Cadera en Flexión $\left(\mathrm{AB}_{\mathrm{F}}\right)$ deben incluirse en cualquier evaluación deportiva músculo-esquelética. La utilización de ambas pruebas permitirá evaluar espe- cíficamente la extensibilidad aductora. Para la realización de ambas pruebas, será necesaria tanto la utilización del goniómetro de rama larga como de un inclinómetro. De manera general, se han encontrado elevados valores de fiabilidad relativa y absoluta en el test $\mathrm{AB}_{\mathrm{PR}}$. Por el contrario, no se han encontrado estudios que analicen la fiabilidad del test $\mathrm{AB}_{\mathrm{F}}$.

\section{Apéndice 1}

\section{Pruebas de valoración}

La prueba de valoración sigue las directrices metodológicas establecidas por la American Academic of Orthopedic Association (1965) y la American Medical Association (2002). Se requiere de un inclinómetro ISOMED (Portland, Oregon) Unilevel con varilla telescópica extensible (Gerhardt, 1994; Gerhardt et al., 2002; Cejudo et al., 2015), un goniómetro metálico de rama larga (Baseline Stainless) y un lumbosant -soporte lumbar para estandarizar la curvatura lumbar- (Santonja, 1995). Previo a cada sesión de valoración, el inclinómetro se calibra a $0^{\circ}$ con la vertical.

Para un mejor entendimiento del procedimiento de evaluación (por ejemplo, instrumentos, posición de los examinadores, posición inicial, posición final), una descripción detallada se muestra en la figura 1 y 2 .

Figura 1. Valoración de la abducción de la cadera con la pierna recta.
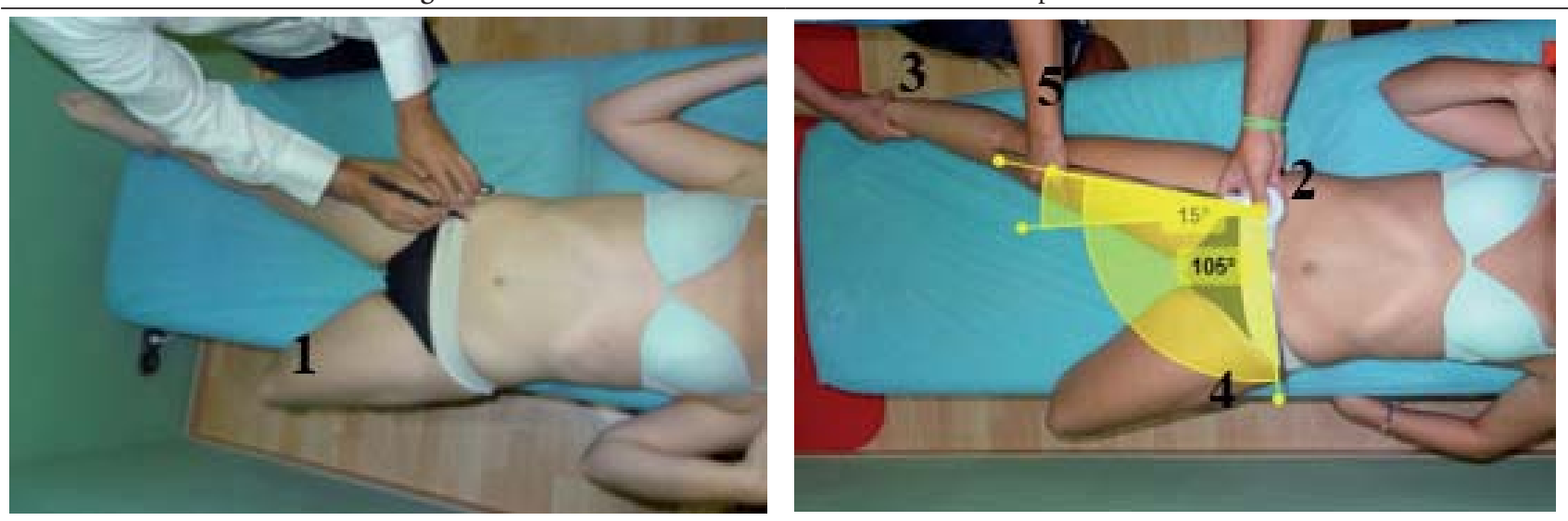

Mide indirectamente la extensibilidad de los aductores (pectíneo, aductor menor, aductor mediano, aductor mayor y el recto interno o gracilis).

1. Posición inicial: El explorado en decúbito supino. La pierna de la extremidad no evaluada se deja colgando por el lateral de la camilla. La extremidad que va a ser valorada se coloca sobre la camilla con la rodilla extendida (figura 3).

2. Colocación del goniómetro: Una rama del goniómetro se coloca uniendo ambas espinas iliacas ántero-superiores y la otra rama se apoya sobre la cara anterior del muslo evaluado siguiendo su bisectriz.

3. Movimiento: El examinador auxiliar realiza de forma lenta y progresiva una abducción de cadera con la rodilla extendida.

4. Estabilización: El examinador auxiliar debe evitar la rotación de la extremidad inferior. La extremidad no explorada cuelga por el lateral de la camilla con la rodilla flexionada.

5. Posición final: movimiento pasivo máximo hasta la "sensación de estiramiento muscular" y/o detención de algún movimiento de compensación. Los posibles movimientos de compensación son la inclinación lateral de la pelvis, la extensión de rodilla de la extremidad no explorada y/o la rotación de la extremidad evaluada.

6. Medición: Se registra el ángulo obtenido entre ambas ramas del goniómetro. 
Figura 2. Valoración de la abducción de la cadera en flexión.

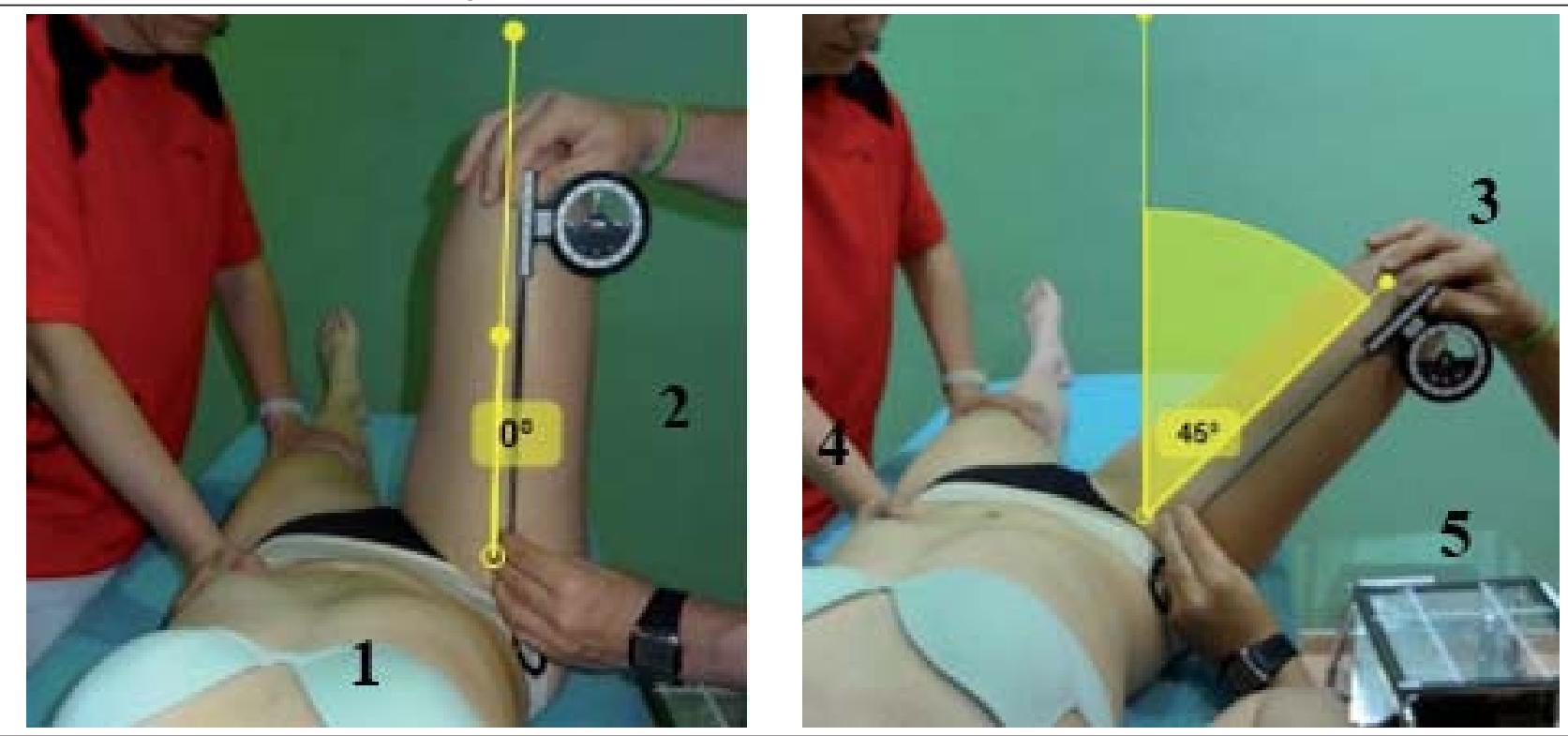

Mide indirectamente la extensibilidad de los aductores monoarticulares (pectíneo, aductor menor, aductor mediano o largo y aductor mayor).

1. Posición inicial: explorado en decúbito supino; la cadera que va a ser valorada se coloca en flexión de $90^{\circ}$ con la rodilla en flexión cómoda.

2. Calibración y colocación del inclinómetro: el evaluador debe calibrar el inclinómetro con la vertical. La varilla telescópica del inclinómetro se coloca sobre la cara anterior del muslo siguiendo su bisectriz.

3. Movimiento: el examinador principal realiza de forma lenta y progresiva una abducción de cadera con la rodilla flexionada.

4. Estabilización: el examinador auxiliar presiona sobre la cara anterior de la hemipelvis contraria para evitar su elevación (rotación).

5. Posición final: movimiento pasivo máximo "sensación de estiramiento muscular" y/o detención por producirse algún movimiento de compensación. Un posible movimiento de compensación es el despegamiento de la hemipelvis contralateral al producirse su rotación horaria o antihoraria.

6. Medición: se registra el ángulo que forma el eje longitudinal anterior del muslo con la vertical.

\section{Bibliografía}

1. Alricsson, M. \& Werner, S. (2004). The effect of pre-season dance training on physical indices and back pain in elite cross-country skiers: a prospective controlled intervention study. British Journal of Sports $\mathrm{Me}$ dicine, 38(2), 148-153.

2. Alricsson, M., Harms-Ringdahl, K., Eriksson, K. \& Werner, S. (2003). The effect of dance training on joint mobility, muscle flexibility, speed and agility in young cross-country skiers-a prospective a controlled intervention study. Scandinavian Journal of Medicine and Science in Sports, $13,273-243$.

3. Alter, M.J. (2004). Los Estiramientos. Barcelona: Paidotribo.

4. American Academy of Orthopaedic Surgeon (1965). Joint Motion: Method of Measuring and Recording. Chicago: Park Ridge.

5. Anderson, K, Strickland, S.M. \& Warren, R. (2001). Hip and Groin Injuries in Athletes. American Orthopaedic Society for Sports Medicine, 29(4), 521-533.

6. Arnason, A., Gudmundsson, Dahl, H.A. \& Johannsson E. (1996). Soccer injuries in Iceland. Scandinavian Journal of Medicine and Science in Sports, 6, 40-45.

7. Arnason, A., Sigurdsson, S. B., Gudmundsson, A., Holme, I., Engebretsen, L. \& Bahr, R. (2004). Risk factors for injuries in football. American Journal of Sports Medicine, 32(1 suppl), 5S-16S.
8. Bishop, D. (2003). Warm Up I. Potential Mechanisms and the Effects of Passive Warm Up on Exercise Performance. Sports Med, 2003; 33 (6): 439-454

9. Bozic, P.R., Pazin, N.R., Berjan, B.B., Planic, N.M. \& Cuk, I.D. (2010). Evaluation of the field tests of flexibility of the lower extremity: reliability and the concurrent and factorial validity. Journal of Strength and Conditioning Research, 24(9), 2523-2531.

10. Bradley, P. \& Portas, M. (2007). The relationship between preseason range of motion and muscle strain injury in elite soccer players. Journal of Strength and Conditioning Research, 21(4), 1155-1159.

11. Calais-Germain B. (2006). Anatomía para el movimiento, Introducción al análisis de las técnicas corporales. Barcelona: La Liebre de Marzo.

12. Cejudo, A., Ayala, F. Sainz de Baranda, P., \& Santonja, F. (2015b). Reliability of two methods of clinical examination of the flexibility of the hip adductor muscles. The International Journal of Sports Physical Therapy, 10(7), 1-8.

13. Cejudo, A., Sainz de Baranda, P., Ayala, F. \& Santonja, F. (2015). Testretest reliability of seven common clinical tests for assessing lower extremity muscle flexibility in futsal and handball players. Physical Therapy in Sport, 16(2), 107-13.

14. Cejudo, A., Sainz de Baranda, P., Ayala, F. y Santonja, F. (2013). Rango 
de movimiento de la extremidad inferior en atletas de duatlón. SporTK, 2(2), 31-40.

15. Cejudo, A., Sainz de Baranda, P., Ayala, F. y Santonja, F. (2014a). Perfil de flexibilidad de la extremidad inferior en jugadores de fútbol sala. Revista Internacional de Medicina y Ciencias de la Actividad Física y el Deporte, 14(55), 509-525.

16. Cejudo, A., Sainz de Baranda, P., Ayala, F. y Santonja, F. (2014b). Perfil de flexibilidad de la extremidad inferior en jugadores sénior de balonmano. Cuadernos de Psicología del Deporte, 14(2), 111-120.

17. Cejudo, A., Sainz de Baranda, P., Ayala, F., Gómez, P. y Santonja, F. (2012). Perfil de flexibilidad óptimo en jugadores de fútbol de $3^{\text {a Divi- }}$ sión Nacional. VII Congreso Internacional de la Asociación Espańola de Ciencias del Deporte.

18. Clarkson, H.M. (2003). Proceso evaluativo músculo-esquelético. Barcelona: Paidotribo.

19. Dallinga, J.M., Benjaminse, A. \& Lemmink K.A.P.M. (2012). Which screening tools can predict injury to the lower extremities in team sports? A systematic review. Sports Medicine, 42, 791-815.

20. Egebretsen, A.H., Myklebust, G., Holme, I., Egebretsen, L. \& Bahr, R. (2010). Intrinsic risk factors for groin injuries among male soccer players: a prospective cohort study. American Journal of Sports Medicine, 38, 2051-2057.

21. Ekstrand, J. \& Gillquist, J. (1982). The frecuency of muscle tightness and injuries in soccer players. American Journal of Sports Medicine, 10(2), 75-78.

22. Ekstrand, J. \& Gillquist, J. (1983). The avoidability of soccer injuries. Internacional Journal Sports Medicine, 4, 124-128.

23. Ekstrand, J. \& Hilding, J. (1999). The incidence and differential diagnosis of acute groin injuries in male soccer players. Scandinavian Journal of Medicine and Science in Sports, 9, 98-103.

24. Ekstrand, J., Wiktorsson, M., Oberg, B. \& Gillquist, J. (1982). Lower extremity goniometric measurements: A study to determine their reliability. Archives of Physical Medicine and Rehabilitation, 63(4), 171-175.

25. Ellenbecker, T.S., Ellenbecker, G.A., Roetert, E.P., Silva, R.T., Keuter, G. \& Sperling, F. (2007). Descriptive profile of hip rotation range of motion in elite tennis players and professional baseball pitchers. American Journal of Sports Medicine, 35(8), 1371-1376.

26. Emery, CA and Meeuwisse, WH. Risk factors for groin injuries in hockey. Med Sci Sports Exerc, 33: 1423-1433, 2001.

27. Fourchet, F., Materne, O., Horobeanu, C., Hudacek, T. \& Buchheit, M. (2013). Reliability of a novel procedure to monitor the flexibility of lower limb muscle groups in highly trained adolescent athletes. Physical Therapy in Sport, 14, 28-34.

28. Fredriksen, H., Dagfinrud, H., Jacobsen, V. \& Maehlum, S. (1997). Passive knee extension test to measure hamstring muscle tightness. Scandinavian Journal of Medicine and Science in Sports, 7, 279-282.

29. Frehill, M.T., Brian, B.G., Archer, K.R., Bancells, R.L., Wilckens, J.H., McFarland E.G. \& Cosgarea, A.J. (2012). Glenohumeral Range of Motion in Major League Pitchers: Changes Over the Playing Season. Journal Athletic Training, 3(1), 97-104.

30. Gannon, L.M. \& Bird, H.A. (1999). The quantification of joint laxity in dancers and gymnasts. Journal of Sports Sciences, 17, 743-750.

31. Gerhardt, J. (1994). Documentation of Joint Motion. Oregon: Isomed.

32. Gerhardt, J., Cocchiarella, L. \& Lea, R. (2002). The Practical Guide to Range of Motion Assessment. Chicago: American Medical Association.

33. Grote, K., Lincoln, T.L. \& Gamble, J.G. (2004). Hip adductor injury in competitive swimmers. American Journal Sports Medicine, 32, 104-108.

34. Hopkins, W.G. (2000). Measures of reliability in sports medicine and science. Sports Medicine, 30(1), 1-15.

35. Irurtia, A., Busquets, A., Carrasco, M., Ferrer, B. \& Marina, M. (2010). Control de la flexibilidad en jóvenes gimnastas de competición mediante el método trigonométrico: un año de seguimiento. Apunts, 45(168), 235-242.
36. Kapandji, A.I. (2007). Fisiología Articular: Miembro Inferior. Madrid: Panamericana.

37. Magee, D.J. (2002). Orthopedic physical assessment, (4th ed.). Philadelphia, Pennsylvania: Saunders Company.

38. Malliaras, P., Cook, J. L. \& Kent, P. (2006). Reduced ankle dorsiflexion range may increase the risk of patellar tendon injury among volleyball players. Journal of Science and Medicine in Sport, 9(4), 304-309.

39. Manning, C. \& Hudson, Z. (2009). Comparison of hip joint range of motion in professional youth and senior team footballers with agematched controls: An indication of early degenerative change?. Physical Therapy in Sport, 10, 25-29.

40. Norkin, C. \& White, J. (2006). Goniometría. Evaluación de la Movilidad Articular. Madrid: Marban.

41. Nussbaumer, S., Leunig, M., Glatthorn, J.F., Stauffacher, S., Gerber, H. \& Maffiuletti, N. A. (2010). Validity and test-retest reliability of manual goniometers for measuring passive hip range of motion in femoroacetabular impingement patients. Musculoskeletal Disorders 11(1), 194-205.

42. Palmer, M.L. \& Epler, M.E. (2002). Fundamentos de las técnicas de la evaluación musculo-esquelética. Barcelona: Paidotribo.

43. Peterson F, Kendall E \& Geise P. (2005). Músculos, Pruebas, funciones y dolor postural. Madrid: Marban.

44. Prather, H., Harris-Hayes, M., Hunt, D.M., Steger-May, K., Mathew, V. \& Clohisy, J.C. (2010). Reliability and agreement of hip range of motion and provocative physical examination tests in asymptomatic volunteers. Journal of injury, function and rehabilitation, 2(10), 888895.

45. Reid, D.C., Burnham, R.S., Saboe, L.A. \& Kushner, S.F. (1987). Lower extremity flexibility patterns in classical ballet dancers and their correlation to lateral hip and knee injuries. American Journal of Sports Medicine, 15(4), 347-352.

46. Sainz de Baranda, P., Cejudo, A., Ayala, F. \& Santonja, F. (2015). Perfil de flexibilidad de la extremidad inferior en jugadoras sénior de fútbol sala. Revista Española de Educación Física y Deportes, 409, 35-48.

47. Steinberg, N., Hershkovitz, I., Peleg, S., Dar, G., Masharawi, Y., Heim, M. \& Siev-Ner, I. (2006). Range of joint movement in female dancers and nondancers aged 8 to 16 years. Americam Orthopaedic Society for Sports Medicine, 34(5), 814-823.

48. Taboadela C, 2007. Goniometría: Una herramienta para la evaluación de las incapacidades laborales. Buenos Aires: Asociart Art.

49. Tyler, T.F., Nicholas, S.J., Campbell, R.J. \& McHugh, M.P. (2001). The association of hip strength and flexibility with the incidence of adductor muscle strains in professional ice hockey players. American Journal Sports Medicine, 29, 124-128.

50. Verrall, G.M., Hamilton, I.A., Slavotinek, J.P., Oakeshott, R.D., Spriggins, A.J., Barnes, P.G. \& Fon, G.T. (2005). Hip joint range of motion reduction in sports-related chronic groin injury diagnosed as pubic bone stress injury. Journal Science Medicine Sport, 8(1), 77-84.

51. Verrall, G.M., Slavotinek, J.P., Barnes, P.G., Esterman, A., Oakeshott, R.D. \& Spriggins, A.J. (2007). Hip joint range of motion restriction precedes athletic chronic groin injury. Journal Science Medicine Sport, 10(6), 463-6.

52. Weppler, C.H. \& Magnusson, S.P. (2010).Increasing Muscle Extensibility: A Matter of Increasing Length or Modifying Sensation?. Physical Therapy, 90(3), 438-449.

53. Witvrouw, E., Bellemans, J., Lysens, R., Danneels, L. \& Cambier, D. (2001). Intrinsic Risk Factors for the Development of Patellar Tendinitis in an Athletic Population A Two-Year Prospective Study. American Journal of Sports Medicine, 29(2), 190-195.

54. Witvrouw, E., Danneels, L., Asselman, P., D’Have, T. \& Cambier, D. (2003). Muscle Flexibility as a Risk Factor for Developing Muscle Injuries in Male Professional Soccer Players. American Journal of Sports Medicine, 31(1), 41-46. 
55. Wong, P \& Hong, Y. Soccer injury in lower extremities (2005). British Journal Sports Medicine, 39, 473-482.

56. Young, S.W., Dakic, J., Stroia, K., Nguyen, M.L., Harris, A.H. \& Safran, M.R. (2014). Hip range of motion and association with injury in female professional tennis players. American Journal of Sports Medicine 42(11), 2654-2658.

57. Zakas, A. (2005). The effect of warming up on the flexibility of adolescent élite tennis players. Journal of Human Movement Studies, 48, 133146.

58. Zakas, A., Galazoulas, C., Zakas, N., Vamvakoudis, E. \& Vergou, A. (2005). The Effect of stretching duration on flexibility during warming up in adolescent soccer players. Physical Training, 48, 133-146.
59. Zakas, A., Grammatikopoulou, M.G., Zakas, N., Zahariadis, P. \& Vamvakoudis, E. (2006). The effect of active warm-up and stretching on the flexibility of adolescent soccer players. Journal of Sports Medicine and Physical Fitness 46(1), 57-61.

60. Zakas, A., Vergou, A., Zakas, N., Grammatikopoulou, M.G. \& Grammatikopoulou, G.T. (2002). Handball match effect on the flexibility of junior handball players. Journal of Human Movement Studies, 43, 321330.

61. Zakas, A., Vergou, M., Grammatikopoulou, N., Sentelidis, T. \& Vamvakoudis, S. (2003). The effect of stretching during warming up on the flexibility of junior handball players. Journal of Sports Medicine and Physical Fitness, 43, 145-149. 